\title{
Caracterización Morfológica del Arco Plantar Longitudinal Medial del Pie en una Población Chilena
}

\author{
Morphological Characterization of the Medial Plantar \\ Longitudinal Arch Foot in a Chilean Population
}

\author{
Sánchez Ramírez, Celso ${ }^{1,2}$
}

SÁNCHEZ, R. C. Caracterización morfológica del arco plantar longitudinal medial del pie en una población Chilena. Int. J. Morphol., 35(1):85-91, 2017.

RESUMEN: El arco plantar longitudinal medial del pie (APLM) es el componente más importante en estática y dinámica de la bóveda plantar. Su altura define pies normales, planos y cavos, estos últimos son factores predisponentes de lesiones. El objetivo de este estudio fue describir y relacionar en una población de estudiantes universitarios chilenos la prevalencia de pies normales, cavos y planos según índice de masa corporal (IMC), sexo y actividad física (AF) semanal y la distribución de asimetrías entre pies. Se estudió a 209 sujetos (128 hombres y 81 mujeres), estudiantes universitarios, cuya edad, estatura, peso e IMC fue de 20,4 años ( $\pm 3,4) ; 1,68 \mathrm{~m}( \pm 0,09)$; $67,6 \mathrm{Kg}( \pm 12,4)$ y $23,77( \pm 3,0)$ respectivamente. Por medio de cuestionario se determinó los hábitos de AF de la muestra. La impresión plantar se obtuvo mediante fotopodoscopía, la cual fue evaluada empleando el Arch Index (AI) de Cavanagh y Rodgers. Los hombres presentan mayor prevalencia de pie plano (31,3\% contra 11,1\%). Hay correlación positiva estadísticamente significativa entre AI e IMC en ambos sexos. Se obtuvo mayor correlación entre AI izquierdo y AF en hombres y mujeres con significancia estadística. El 31,25 \% de los hombres y el 37,04 \% de las mujeres presentaron asimetrías en APLM. Hombres con IMC normal tienen 64,20\% de pies asimétricos. Las mujeres que practican entre 6 y 10 horas de AF a la semana presentan 50,98\% de pies asimétricos. El IMC se relaciona con una disminución de la altura del APLM, se sugiere el control de esta variable en sujetos con diagnóstico de pie plano flexible y pie plano valgo. La práctica de AF se relaciona con aumento de la altura del APLM en hombres y mujeres y se sabe que también contribuye a disminuir el IMC, actuando en concordancia hacia estas dos variables.

PALABRAS ClAVE: Arco plantar; Pie plano; Pie Cavo; Arch Index.

\section{INTRODUCCIÓN}

El ser humano es un mamífero plantígrado, pero a diferencia de otros, la configuración morfológica de la planta del pie, se ha descrito como medio domo o bóveda con tres puntos de apoyo principales y que, al juntar ambos pies, se forma una bóveda completa (Snell, 2002). El arco plantar longitudinal medial (APLM) se ha reconocido como el principal y más importante componente, tanto en la estática como en la dinámica de la bóveda plantar, debido a que es el que posee mayor longitud y altura y porque es la estructura con mayor incidencia en la absorción, acumulación y devolución de fuerzas gravitacionales durante los apoyos (Kapandji, 2010).

El APLM debe su morfología a la constitución anatómica de los huesos que lo componen, a la mantención pasiva que prestan estructuras ligamentosas, y a la función activa de músculos intrínsecos y extrínsecos del pie (Viladot, 2000).
Según la altura del arco, tradicionalmente se han identificado tres tipologías de pie: normal, plano y cavo. Pies planos y cavos se han reconocido como factores predisponentes de lesiones, sobre todo durante la práctica de actividad física y deportiva (Razeghi \& Batt, 2002; Shiang et al., 1998; Kulthanan et al., 2004), ya que según la altura del arco, se modifican las áreas de apoyo plantar y con eso también el patrón de distribución de presiones. Un pie plano centra las presiones sobre el hueso navicular, factor predisponente a fracturas por estrés (Chuckpaiwong et al., 2008). Un arco elevado también tiene mayor relación con lesiones, provocadas principalmente por el aumento de presión sobre los puntos de apoyo y por el aumento de tensión en la fascia plantar (Burns et al., 2005; Hreljac et al., 2000)

La altura del APLM ha sido evaluado de diversas

\footnotetext{
${ }^{1}$ Laboratorio de Ciencias de la Actividad Física - Universidad de Santiago de Chile, Santiago, Chile.

${ }^{2}$ Estudiante de Doctorado en Investigación Sociosanitaria y de la Actividad Física - Universidad de Castilla-La Mancha, España.

Estudio financiado por la Dirección de Investigación Científica y Tecnológica (Dicyt) de la Universidad de Santiago de Chile, Santiago, Chile. código 021687SR_OP.
} 
maneras, no obstante, el método más empleado ha sido el estudio de la huella plantar debido a la facilidad que supone su obtención, como por los buenos indicadores de confiabilidad y validez que presenta (Cavanagh \& Rodgers, 1987). La obtención de la huella plantar presenta altas correlaciones con métodos radiográficos (Villarroya et al., 2009) y se utiliza incluso como estándar de oro para la validación de equipos más sofisticados como escáneres o baropodógrafos (Urry \& Wearing, 2005).

La altura del APLM ha sido estudiada empleando diversos métodos y en diversas poblaciones. Sus resultados son expresados generalmente según distribución de edad, sexo, índice de masa corporal (IMC) y actividad física (AF) (Bayat et al., 2015).

A pesar que el conocimiento de la altura del APLM en una población permite comprender de mejor manera el origen de algunas lesiones que afectan al pie, tobillo y rodilla, y que es un insumo de utilidad para la elaboración de calzado ergonómico, en Chile no se han hecho estudios orientados a la evaluación objetiva de esta variable. Cabe hacer mención de solo un estudio publicado, que emplea una metodología observacional no cuantitativa en una población de escolares de la ciudad de Arica (Espinoza-Navarro et al., 2013).

Por lo anterior, el objetivo de este estudio es describir por primera vez en una población de estudiantes universitarios chilenos la prevalencia de pies normales, cavos y planos según IMC, sexo y horas de práctica de AF semanal y la distribución de asimetrías entre pies de un mismo individuo.

\section{MATERIAL Y MÉTODO}

Este es un estudio del tipo descriptivo exploratorio. La muestra corresponde a 209 sujetos de ambos sexos, obtenidos aleatoriamente de una población de estudiantes de la Universidad de Santiago de Chile, cuya edad, estatura y peso fue de 20,4 años $( \pm 3,4), 1,68 \mathrm{~m}( \pm 0,09)$ y $67,6 \mathrm{Kg}$ $( \pm 12,4)$ respectivamente. El IMC promedio fue de 23,77 $( \pm 3,0)$. La muestra estuvo compuesta por 128 hombres y 81 mujeres, quienes accedieron de forma voluntaria a participar del estudio, acreditando esto por medio de la firma del consentimiento informado redactado según las directrices del Comité de Ética de la Universidad de Santiago de Chile. Junto con la firma del consentimiento, los sujetos llenaron un cuestionario en donde se les consultó por la cantidad de horas de práctica de AF que realizaban a la semana y por la presencia de lesiones en tobillo y/o pie. Se excluyó a todos los sujetos que presentaban lesión en etapa aguda.

Las evaluaciones se efectuaron durante un período de tiempo de 2 meses y siempre en un mismo día y horario de la semana.

La obtención del peso corporal se efectuó con los sujetos vestidos en ropa interior, en una balanza digital marca Tanita, Modelo HD 314. La estatura se registró por medio de una cinta métrica adosada a la pared. Con estos valores se obtuvo el IMC (Peso Corporal / Estatura²).

Luego, los sujetos se subieron a un podoscopio de metal con cristal sólido. Previamente, mantuvieron reposo sentado mínimo 30 minutos antes de las mediciones, a fin de no producir modificaciones en la forma del pie (Jiménez et al., 2011).

Basado en el protocolo de Ribeiro et al. (2006), sobre el podoscopio los sujetos mantuvieron durante un minuto postura en bipedestación con brazos relajados a los costados del cuerpo y la vista hacia el horizonte. Los pies se mantuvieron separados $10 \mathrm{~cm}$, distancia demarcada sobre el cristal del podoscopio. Luego de eso, se procedió a fotografiar la huella plantar con una cámara digital marca Samsung modelo ST65 de 14,2 megapixeles, sin zoom y a una distancia focal de $43 \mathrm{~cm}$ directamente sobre el vidrio.

El análisis de la fotografía de ambas impresiones plantares se efectuó empleando el software específico de cálculo de áreas plantares AreaCalc, desarrollado por Elvira et al. (2008). La clasificación del APLM se realizó mediante el Arch Index (AI) (Cavanagh \& Rodgers), que emplea la siguiente fórmula:

$$
\mathrm{AI}=\mathrm{B} /(\mathrm{A}+\mathrm{B}+\mathrm{C})
$$

Donde A corresponde al área del retropié, B es el área del mediopié y $\mathrm{C}$ corresponde al área del antepié (Fig. 1).

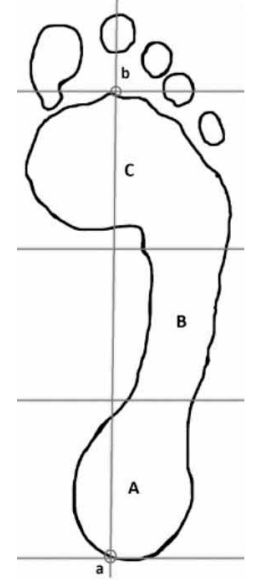

Fig. 1. Obtención del Índice de Cavanagh.
Los valores usados para clasificar la altura del arco fueron los recomendados por el creador del método: Pie cavo AI $\leq 0,21$; Pie normal 0,21 < AI < 0,26 y Pie plano $\mathrm{AI} \geq 0,26$.

El análisis estadístico se efectuó con software GraphPad Prism, versión 6. Se aplicó estadígrafos de tendencia central para las variables de edad, peso, estatura, IMC y AI de ambos pies. Se efectuó análisis de normalidad de AI de ambos pies usando la prue- 
ba estadística de Kolmogórov-Smirnov. La comparación entre pies derechos e izquierdos permitió establecer asimetría de pies, calculada mediante el uso de la prueba estadística Wilcoxon para muestras relacionadas. Además, se aplicó el coeficiente $r$ de Spearman para establecer correlaciones entre las variables de AI, IMC y AF. Se consideró p $<0,05$ como valor de significancia estadística.

\section{RESULTADOS}

La Tabla I refleja los valores de edad, estatura, peso, IMC y AI de la muestra estudiada. La edad de la muestra ronda los 20 años. Hombres y mujeres muestran una media de IMC equivalente a peso normal. La media de AI se encuentra dentro del rango de pie normal, no obstante, en mujeres los valores se acercan al límite inferior, cercano a pie cavo.

La Tabla II clasifica a la muestra estudiada según valores de IMC y de AF semanal. Las mujeres presentaron mayor porcentaje de sujetos con peso normal que los hombres $(81,5 \%$ contra 63,3\%). Asimismo, los hombres muestran mayor porcentaje de sujetos con sobrepeso y obesidad sumando $35,9 \%$. Las mujeres en cambio, presentaron 18,5 $\%$ de sujetos con sobrepeso. No hubo mujeres con bajo peso. En relación a la cantidad de horas de AF que los sujetos practicaban a la semana, se observa en hombres y mujeres una distribución mayor de sujetos que practicaban entre 6 a 10 horas a la semana. Hubo mayor porcentaje de hombres

Tabla I. Caracterización de la muestra estudiada.

\begin{tabular}{lrr}
\hline & HOMBRES & MUJERES \\
& $\mathbf{n}=\mathbf{1 2 8}$ & $\mathbf{n = 8 1}$ \\
\hline Edad (años) & $20,20( \pm 3,66)$ & $20,89( \pm 3,00)$ \\
Estatura (m) & $1,73( \pm 0,07)$ & $1,60( \pm 0,07)$ \\
Peso (kg) & $73,22( \pm 11,80)$ & $58,83( \pm 7,20)$ \\
IMC & $24,36( \pm 3,30)$ & $22,84( \pm 2,15)$ \\
AI Derecho & $0,23( \pm 0,05)$ & $0,21( \pm 0,06)$ \\
AI Izquierdo & $0,22( \pm 0,06)$ & $0,22( \pm 0,06)$ \\
\hline
\end{tabular}

Tabla II. Distribución de la muestra según sexo, IMC y hábitos de práctica de AF.

\begin{tabular}{lcc}
\hline & HOMBRES \% & MUJERES \% \\
\hline IMC & & \\
Bajo Peso & 0,8 & 0,0 \\
Peso Normal & 63,3 & 81,5 \\
Sobrepeso & 28,9 & 18,5 \\
Obesidad & 7,0 & 0,0 \\
Act. física / semana & & \\
$<6$ hrs & 38,3 & 33,3 \\
6 a 10 hrs & 52,3 & 63,0 \\
$>10$ hrs & 9,4 & 3,7 \\
\hline
\end{tabular}

que practicaban menos de 6 horas y más de 10 horas respecto a las mujeres.

La Tabla III muestra la distribución del tipo de pie según la altura del arco en hombres y mujeres y según pie derecho e izquierdo. En todos los casos, el mayor porcentaje fue de pies normales, no obstante, las mujeres mostraron valores mayores de frecuencia de este tipo de pie que los hombres. Se observa que los hombres muestran mayor porcentaje de pies planos que las mujeres, con valores que rondan el $30 \%$. Las mujeres en cambio muestran $11,1 \%$ y 16 $\%$ en pies derechos e izquierdos respectivamente. En cuanto a la distribución de pies cavos, es posible observar que hombres y mujeres presentan valores similares, sin embargo, los hombres siempre muestran porcentajes menores que las mujeres.

Los hombres mostraron una distribución más uniforme de tipo de pie que las mujeres. Aun así, los pies derechos de hombres demostraron ser mayormente planos y normales. Los pies izquierdos de hombres en cambio, mostraron mayor distribución de arco alto. Las mujeres revelaron pies derechos con mayor distribución de pies normales (58 $\%)$. Los pies izquierdos de mujeres mostraron mayores porcentajes de pies planos (16\% contra $11,1 \%)$ y cavos $(38,3$ $\%$ contra $30,9 \%$ ).

La Tabla IV ilustra la distribución de tipo de pie según el IMC de los sujetos. En ella se puede ver que sujetos clasificados como normo peso presentan en general mayores porcentajes de pies normales, siguiendo la misma tendencia observada en la Tabla III. No obstante, llama la atención que en pies izquierdos de hombres hay una mayor distribución de pies cavos $(39,5 \%)$, superando la distribución de pies planos y normales. En los normo peso, además, es notorio que los pies izquierdos son más cavos que los pies derechos, en hombres $(39,5 \%$ contra $32,1 \%)$ y mujeres $(40,9 \%$ contra $33,3 \%)$.

En los hombres con sobrepeso, aumenta el porcentaje de pies planos y pies normales, disminuyendo el porcentaje de pies cavos. Las mujeres en cambio, aumentan el porcentaje de pies normales con valores de $66,7 \%$ para pies derechos y 60,0 \% para pies izquierdos, y disminuyen los porcentajes de pies cavos. Asimismo, aumenta la distribución de pies planos derechos (10,6\% a 13,3\%).

Tabla III. Distribución de la muestra según sexo y altura del APLM.

\begin{tabular}{lcccc}
\hline & \multicolumn{2}{c}{ HOMBRES } & \multicolumn{2}{c}{ MUJERES } \\
& Pie Der. \% & Pie Izq. \% & Pie Der. \% & Pie Izq. \% \\
\hline Plano & 31,3 & 28,9 & 11,1 & 16,0 \\
Normal & 39,8 & 35,9 & 58,0 & 45,7 \\
Cavo & 28,9 & 35,2 & 30,9 & 38,3 \\
\hline
\end{tabular}


Los hombres obesos aumentan súbitamente la proporción de pies con arco bajo, mostrando un 55,6 \% para ambos pies. Asimismo, disminuye la frecuencia de pies normales $(11,1$ $\%)$ pero aumenta el porcentaje de pies cavos también $(33,3 \%)$.

Con fines descriptivos, la muestra estudiada se clasificó en tres categorías de acuerdo a la cantidad de horas de $\mathrm{AF}$ semanal. La tabla $\mathrm{V}$ muestra que los hombres que practicaron menos de 6 horas de AF a la semana, presentan una distribución homogénea de tipo de pie. Los pies derechos mostraron mayor distribución de pies planos y normales, mientras los pies izquierdos presentan mayor porcentaje de pies normales. Las mujeres en cambio, muestran mayor distribución de pies normales ( $63 \%$ en pie derecho y $59 \%$ en pie izquierdo), observándose bajos valores de pie plano y de pie cavo respecto a los hombres.

Los hombres que practicaban de 6 a 10 horas de AF a la semana, presentan un aumento en el porcentaje de pies cavos izquierdos $(38,8 \%)$ y de pies normales derechos $(38,8$ $\%)$. Las mujeres en tanto, muestran pies derechos con alta distribución de pies normales $(56,9 \%)$ y pies izquierdos con mayor distribución de pies cavos $(45,1 \%)$. Además, presentan baja distribución de pies planos.

Los hombres que practicaban más de 10 horas de $\mathrm{AF}$ a la semana, se caracterizaron por presentar mayores porcentajes de pies normales y bajos porcentajes de pies planos. Sin embargo, llama la atención la diferencia en la distribución de pies cavos entre pie derecho $(16,7 \%)$ y pie izquierdo $(41,7 \%)$. Las mujeres con más práctica de $\mathrm{AF}$ aumentaron el porcentaje de pies cavos derechos e izquierdos $(66,7 \%)$ y nulos casos con pie plano.

Tabla IV. Distribución de altura del APLM según IMC en ambos sexos.

\begin{tabular}{lcccc}
\hline & $\begin{array}{c}\text { HOMBRES } \\
\text { Pie Der. \% }\end{array}$ & \multicolumn{3}{c}{ MUJERES } \\
& Pie Izq. \% & Pie Der. \% & Pie Izq. \% \\
\hline Bajo Peso & 0,0 & 0,0 & 0,0 & 0,0 \\
Plano & 100,0 & 100,0 & 0,0 & 0,0 \\
Normal & 0,0 & 0,0 & 0,0 & 0,0 \\
Cavo & & & & \\
Peso Normal & 28,4 & 24,7 & 10,6 & 16,7 \\
Plano & 39,5 & 35,8 & 56,1 & 42,4 \\
Normal & 32,1 & 39,5 & 33,3 & 40,9 \\
Cavo & & & & \\
Sobrepeso & 32,4 & 32,4 & 13,3 & 13,3 \\
Plano & 45,9 & 40,5 & 66,7 & 60,0 \\
Normal & 21,6 & 27,0 & 20,0 & 26,7 \\
Cavo & & & & \\
Obesidad & 55,6 & 55,6 & 0,0 & 0,0 \\
Plano & 11,1 & 11,1 & 0,0 & 0,0 \\
Normal & 33,3 & 33,3 & 0,0 & 0,0 \\
Cavo & & & & \\
\hline
\end{tabular}

Tabla V. Distribución de altura del APLM según AF en ambos sexos.

\begin{tabular}{lcccc}
\hline & \multicolumn{2}{c}{ HOMBRES } & \multicolumn{2}{c}{ MUJERES } \\
& Pie Der. \% & Pie Izq. \% & Pie Der. \% & Pie Izq. \% \\
\hline < 6 hrs & & & & \\
Plano & 34,7 & 32,7 & 11,1 & 18,5 \\
Normal & 34,7 & 38,8 & 63,0 & 59,3 \\
Cavo & 30,6 & 28,6 & 25,9 & 22,2 \\
6 a 10 hrs & & & & \\
Plano & 31,3 & 29,9 & 11,8 & 15,7 \\
Normal & 38,8 & 31,3 & 56,9 & 39,2 \\
Cavo & 29,9 & 38,8 & 31,4 & 45,1 \\
$>$ 10 hrs & & & & \\
Plano & 16,7 & 8,3 & 0,0 & 0,0 \\
Normal & 66,7 & 50,0 & 33,3 & 33,3 \\
Cavo & 16,7 & 41,7 & 66,7 & 66,7 \\
\hline
\end{tabular}

Las Tablas VI y VII muestran el análisis de correlaciones, donde se observa que hubo valores bajos de correlación entre AI y IMC pero estadísticamente significativos, tanto en hombres como mujeres. Se establece así que a mayor IMC, mayor asociación a pies planos. Asimismo, se obtuvo mayor correlación entre AI izquierdo y AF en hombres y mujeres con significancia estadística.

Tabla VI. Correlaciones en hombres.

\begin{tabular}{lll}
\hline & \multicolumn{2}{c}{ Hombres } \\
& \multicolumn{1}{c}{ IMC } & \multicolumn{1}{c}{ AF } \\
\hline AI Derecho & $\mathrm{r}=0.1790$ & $\mathrm{r}=-0.1185$ \\
& $\mathrm{p}=0.020^{*}$ & $\mathrm{p}=0.0914$ \\
AI Izquierdo & $\mathrm{r}=0.1704$ & $\mathrm{r}=-0.1720$ \\
& $\mathrm{p}=0.0272 *$ & $\mathrm{p}=0.0261^{*}$
\end{tabular}

A fin de establecer asimetría entre pies derechos e izquierdos, se comparó AI derecho con AI izquierdo y se obtuvo que los hombres presentan diferencias significativas $\left(\mathrm{p}=0,0002^{* *}\right)$. Al contrario, las mujeres no presentan diferencias $(\mathrm{p}=0,8986)$. A pesar de lo anterior, al revisar las frecuencias en el total de la muestra, se observó que el $31,25 \%$ de los hombres y el $37,04 \%$ de las mujeres presentaron asimetrías en la altura del arco entre pies derechos e izquierdos.

La Tabla VIII muestra la distribución de porcentaje de asimetría entre pie derecho e izquierdo en los sujetos según IMC y AF. Llama la atención el alto porcentaje de pies asimétricos en hombres con peso normal $(64,2 \%)$. En mujeres normo peso y con sobrepeso se registran valores de $37,88 \%$ y $33,33 \%$ respectivamente.

Los hombres que practican menos de 6 horas y más de 10 horas de AF a la semana presentan 34,69\% y 33,33 $\%$ de sujetos con pies asimétricos. Las mujeres que practican entre 6 y 10 horas de AF a la semana presentan 50,98 $\%$ de pies asimétricos. 
Tabla VII. Correlaciones en mujeres.

\begin{tabular}{lll}
\hline & \multicolumn{2}{c}{ Mujeres } \\
& \multicolumn{1}{c}{ IMC } & \multicolumn{1}{c}{ AF } \\
\hline AI Derecho & $\mathrm{r}=0,2108$ & $\mathrm{r}=-0,1495$ \\
& $\mathrm{p}=0,0295^{*}$ & $\mathrm{p}=0,0915$ \\
AI Izquierdo & $\mathrm{r}=0,2363$ & $\mathrm{r}=-0,2841$ \\
& $\mathrm{p}=0,0168^{*}$ & $\mathrm{p}=0,0051^{*}$ \\
\hline
\end{tabular}

Tabla VIII. Distribución de asimetrías de pie según sexo, IMC y AF.

\begin{tabular}{lcc}
\hline & $\begin{array}{c}\text { HOMBRES } \\
\text { Asimetría \% }\end{array}$ & $\begin{array}{c}\text { MUJERES } \\
\text { Asimetría } \%\end{array}$ \\
\hline I MC & & \\
B ajo Peso & 0,00 & 0,00 \\
Peso Normal & 64,20 & 37,88 \\
Sobrepeso & 37,84 & 33,33 \\
Obesidad & 0,00 & 0,00 \\
AF & & \\
$<6$ hrs & 34,69 & 40,74 \\
6 a 10 hrs & 28,36 & 50,98 \\
$>10$ hrs & 33,33 & 0,00 \\
\hline
\end{tabular}

\section{DISCUSIÓN}

La morfología del APLM condiciona la funcionalidad del pie, hecho que se reafirma aún más durante la práctica de ejercicio físico. Los resultados de esta variable se presentaron según sexo, IMC y horas de práctica de AF semanal de los sujetos.

Antes de contrastar los resultados con lo que existe en la literatura, cabe hacer notar los pocos estudios efectuados en poblaciones adultas universitarias, siendo mucho más frecuentes las investigaciones en poblaciones infantiles (Bhoir et al., 2014).

La media de AI en el caso de los hombres clasificaba dentro de la categoría de arco normal, sin embargo, se acercaba más al valor de pie plano, como lo reportado por Delgado et al. (2012). Las mujeres por su parte, reportan valores de AI dentro de la clasificación de arco normal pero cercano al límite con pie cavo. En la literatura es frecuente encontrar que las mujeres presentan mayor tendencia al pie cavo, como lo informado por Gómez et al. (2009) quienes encuentran en mujeres deportistas una alta prevalencia de pie cavo $(69,81 \%)$, aunque empleando otra metodología de análisis.

Igbigbi et al. (2002), se refiere a 5 estudios de prevalencias de pies planos en poblaciones originarias de Malawi, Estados Unidos, Uganda, Turquía y Nigeria, repor- tando valores de $24,26 \%, 25 \%, 3,88 \%, 3 \%$ y $2,22 \%$ respectivamente, valores por debajo de los cerca de $30 \%$ reportados en hombres de este estudio. Sólo los dos primeros estudios emplearon AI como método de evaluación y son los que presentan valores más cercanos a los reportados en esta muestra.

Como era de esperarse, el pie con APLM normal fue el más frecuente en hombres y mujeres como lo reportado por Abolarin et al. (2011) y Choi et al., (2014). No obstante, mientras estos autores reportan valores de $86,9 \%$ y 91,69 $\%$ respectivamente, en este estudio los porcentajes registrados fueron más bajos en hombres y mujeres. Cabe destacar que los autores citados evaluaron en una población del suroeste de Nigeria y en la tribu Maasai en territorios de Kenia y Tanzania y no emplearon AI como método de evaluación.

La mayor prevalencia de pies planos en hombres y de pies cavos en mujeres, puede relacionarse con el IMC, tal como lo indican los estudios de Espinoza-Navarro et al., y Villarroya et al. Se encontró correlación con significancia estadística entre AI e IMC, lo cual implica que, a mayor peso corporal más bajo el arco, datos concordantes con lo afirmado por Wearing et al. (2012). No sería coincidencia entonces que las mujeres presentan valores de IMC más bajo y mayor prevalencia de pie cavo. Lo anterior se verifica además en el análisis de frecuencias, donde se observa que a medida que suben los valores de IMC, suben también los porcentajes de aparición de pie plano, sobretodo en hombres.

El grueso de la muestra practicaba entre 6 y 10 horas de AF a la semana en hombres y mujeres. Se observó una tendencia que indica que a mayor cantidad de AF, el APLM del pie izquierdo se va elevando. Se observó, que los hombres que practicaban más actividad física presentaron mayor proporción de pies normales; y las mujeres mayor proporción de pies cavos, como lo que indica Gómez et al., (2009).

Al igual que en esta investigación, Kulthanan et al. reporta diferencias significativas entre pie derecho e izquierdo en una muestra de deportistas y sujetos sedentarios, pero no entrega porcentajes de distribución. En este trabajo, alrededor de un tercio de hombres y mujeres tienen pies asimétricos, porcentaje que se contrasta con lo reportado por Ridola et al. (2001), quien encuentra que $22 \%$ de sujetos con incongruencia entre pie derecho e izquierdo. Debido a la gran distribución de pies asimétricos en mujeres que practicaban entre 6 a 10 horas de AF, se presenta como interrogante la asociación de la práctica de AF y/o deportiva como un factor de origen de la asimetría de pies, debido al aumento de presiones sobre el pie de apoyo por ejemplo durante la práctica de fútbol o de deportes de combate. 
El hecho de tener pies con distinta tipología de APLM, podría provocar desalineaciones músculo esqueléticas que podrían ser un factor predisponente a la aparición de lesiones, sobretodo en sujetos que practican actividad física de manera regular. No obstante, se sugiere investigar esta relación empleando métodos experimentales. Asimismo, se plantea la necesidad que las empresas fabricantes de calzado deportivo tomen en consideración las características morfológicas de sus clientes finales, incluyendo modelos que tengan patrones de asimetría presentes en la población objetivo.

De esta investigación se concluye que el aumento del IMC se relaciona con una disminución de la altura del
APLM, situación predisponente de alteraciones mayores en el aparato locomotor. Se sugiere el control de esta variable en sujetos con diagnóstico de pie plano flexible y pie plano valgo. Relacionado a lo anterior, la práctica de $\mathrm{AF}$ se relaciona con aumento de la altura del APLM en hombres y mujeres y se sabe que también contribuye a disminuir el IMC, actuando en concordancia hacia estas dos variables.

Se sugiere continuar estudiando el APLM en poblaciones de distintos grupos de edad, así como en grupos cuya actividad laboral se relaciona con mayor carga sobre los pies, tales como profesionales de la salud que cumplen turnos, deportistas o trabajadores que deben transportar cargas pesadas.

SÁNCHEZ, R. C. Morphological characterization of the medial plantar longitudinal arch foot in a Chilean population. Int. J. Morphol., 35(1):85-91, 2017.

SUMMARY: The (MPLA) is the most important component in statics and dynamics of the vault plant. Its height defines normal, flat and cavus feet, which are predisposing factors for injuries. The objective of this research was to describe and relate in a population of Chilean university students the prevalence of normal, cavus and flat feet according to body-mass index (BMI), sex, and weekly physical activity (PA) and the distribution of asymmetries between feet. 209 subject were studied (128 men and 81 women), university students, whose age, height, weight and BMI was of 20.4 years $( \pm 3.4) ; 1,68 \mathrm{~m}( \pm 0.09) ; 67.6 \mathrm{~kg}( \pm 12.4)$ and $23.77( \pm 3.0)$ respectively. The PAs habits of the sample were determined by means of questionnaire. The footprint is obtained by photo-podoscopy, which was evaluated by the Arch Index (AI) of Cavanagh and Rodgers. Men have higher prevalence of flat foot $(31.3 \%$ against $11.1 \%)$. There are both statistically significant positive correlations between AI and BMI in both sexes. Greater correlation between left AI and PA in both men and women was obtained with statistical significance. The $31.25 \%$ of men and $37.04 \%$ of women presented asymmetries in MPLA. Men with normal BMI have $64.20 \%$ of asymmetric feet. Women that practice between 6 and 10 hours of PA a week presented $50.98 \%$ of asymmetric feet. The BMI is related to a decrease of the height of MPLA, suggesting the control of this variable in subjects with flexible flat foot and valgus flat foot diagnosis. The practice of PA is related to the height of the MPLA increased in men and women and it is known that it also helps to reduce BMI, acting in accordance to these two variables.

\section{KEY WORDS: Arch of the foot; Flat foot; Cavus foot; Arch index.}

\section{REFERENCIAS BIBLIOGRÁFICAS}

Abolarin, T.; Aiyegbusi, A.; Tella, A. \& Akinbo, S. Predictive factors for flatfoot: The role of age and footwear in children in urban and rural communities in South West Nigeria. Foot (Edinb.), 21(4):188-92, 2011.

Bayat, P. D.; Ahmadi, M. M.; Moradi, M.; Ghanbari, A. Effects of anthropometric factors on foot size in normal preschool children of Iran. Int. J. Morphol., 33(3):1141-5, 2015.

Bhoir, T.; Anap, D. B. \& Diwate, A. Prevalence of flat foot among 18-25 years old physiotherapy students: Cross sectional study. Indian J. Basic Appl. Med. Res., 3(4):272-8, 2014.

Burns, J.; Keenan, A. M. \& Redmond, A. Foot type and overuse injury in triathletes. J. Am. Podiatr. Med. Assoc., 95(3):235-41, 2005.

Cavanagh, P. R. \& Rodgers, M. M. The arch index: a useful measure from footprints. J. Biomech., 20(5):547-51, 1987.

Choi, J. Y.; Suh, J. S. \& Seo, L. Salient features of the Maasai foot: analysis of 1,096 Maasai subjects. Clin. Orthop. Surg., 6(4):410-9, 2014.

Chuckpaiwong, B.; Nunley, J. A.; Mall, N. A. \& Queen, R. M. The effect of foot type on in-shoe plantar pressure during walking and running. Gait Posture, 28(3):405-11, 2008.

Delgado-Abellán, L.; Aguado, X.; Jiménez-Ormeño, E.; Mecerreyes, L. \& Alegre, L. M. Efectos del ejercicio continuo e intermitente sobre la huella plantar. Arch. Med. Deporte, 29(148):601-8, 2012.

Elvira, J. L.; Vera-García, F. J. \& Meana, M. Subtalar joint kinematic correlations with footprint arch index in race walkers. J. Sports Med. Phys. Fitness, 48(2):225-34, 2008.

Espinoza-Navarro, O.; Olivares Urquieta, M.; Palacios Navarrete, P. \& Robles Flores, N. Prevalence of foot anomalies in schoolchildren between 6 and 12 years old of elementary education from Arica-Chile. Int. J. Morphol., 31(1):162-8, 2013.

Hreljac, A.; Marshall, R. N. \& Hume, P. A. Evaluation of lower extremity overuse injury potential in runners. Med. Sci. Sports Exerc., 32(9):163541, 2000.

Igbigbi, P. S. \& Msamati, B. C. The footprint ratio as a predictor of pes planus: a study of indigenous Malawians. J. Foot Ankle Surg., 41(6):3947, 2002.

Jimenez-Ormeño, E.; Aguado, X.; Delgado-Abellan, L.; Mecerreyes, L. \& Alegre, L. M. Changes in footprint with resistance exercise. Int. J. Sports Med., 32(8):623-8, 2011.

Kapandji, I. A. Fisiología Articular. Tomo 2. 6a ed. Madrid, Médica Panamericana, 2010.

Kulthanan, T.; Techakampuch, S. \& Bed, N. D. A study of footprints in 
athletes and non-athletic people. J. Med. Assoc. Thai., 87(7):788-93, 2004.

Razeghi, M. \& Batt, M. E. Foot type classification: a critical review of current methods. Gait Posture, 15(3):282-91, 2002.

Ribeiro, A. P.; Trombini-Souza, F..; Iunes, D. H. \& Monte-Raso, V. V. Confiabilidade inter e intra-examinador da fotopodometria e intra-examinador da fotopodoscopia. Bras. J. Phys. Ther, 10(4):435-9, 2006.

Ridola, C.; Palma, A.; Cappello, F.; Gravante, G.; Russo, G.; Truglio, G.; Pomara, F. \& Amato, G. Symmetry of healthy adult feet: role of orthostatic footprint at computerized baropodometry and of digital formula. Ital. J. Anat. Embryol., 106(2):99-112, 2001.

Shiang, T. Y.; Lee, S. H.; Lee, S. J. \& Chu, W. C. Evaluating different footprint parameters as a predictor of arch height. I. E. E. E. Eng. Med. Biol. Mag., 17(6):62-6, 1998.

Snell, R. Anatomía Clínica Para Estudiantes de Medicina. $6^{a}$ ed. Ciudad de México, Mc Graw-Hill/Interamericana, 2002.

Urry, S. R. \& Wearing, S. C. Arch indexes from ink footprints and pressure platforms are different. Foot, 15(2):68-73, 2005.

Viladot, A. Quince lecciones sobre Patología del Pie. Barcelona, SpringerVerlag Ibérica, 2000

Villarroya, M. A.; Esquivel, J. M.; Tomás, C.; Moreno, L. A.; Buenafé, A. \& Bueno, G. Assessment of the medial longitudinal arch in children and adolescents with obesity: footprints and radiographic study. Eur. $J$. Pediatr., 168(5):559-67, 2009.

Wearing, S. C.; Grigg, N. L.; Lau, H. C. \& Smeathers, J. E. Footprintbased estimates of arch structure are confounded by body composition in adults. J. Orthop. Res., 30(8):1351-4, 2012.

\author{
Dirección para correspondencia: \\ Celso A. Sánchez Ramírez \\ Académico \\ Facultad de Ciencias Médicas \\ Escuela de Ciencias de la Actividad Física, el Deporte y la \\ Salud \\ Universidad de Santiago de Chile - USACH \\ Santiago \\ CHILE
}

Email:celso.sanchez@usach.cl

Recibido : 15-10-2016

Aceptado: 24-11-2016 